\title{
À propos du tracé directeur de l'instrument à corde
}

Luc Breton

\section{(2) OpenEdition}

Journals

Édition électronique

URL : http://journals.openedition.org/ethnomusicologie/2344

ISSN : 2235-7688

Éditeur

ADEM - Ateliers d'ethnomusicologie

Édition imprimée

Date de publication : 1 janvier 1989

Pagination : 217-233

ISBN : 2-8257-0178-5

ISSN : 1662-372X

\section{Référence électronique}

Luc Breton, «À propos du tracé directeur de l'instrument à corde », Cahiers d'ethnomusicologie [En ligne], 2 | 1989, mis en ligne le 15 septembre 2011, consulté le 01 mai 2019. URL : http:// journals.openedition.org/ethnomusicologie/2344 


\title{
À PROPOS DU TRACÉ DIRECTEUR DE L'INSTRUMENT À CORDE
}

\author{
Luc Breton
}

\section{Le tracé directeur: une conception cosmologique}

L'appréciation d'un instrument à corde repose actuellement sur divers critères, comme sa valeur historique, ses qualités sonores, son état de conservation, sa beauté ou ses commodités de jeu.

Aux yeux du luthier qui travaille selon des conceptions traditionnelles, toutes ces qualités ne découlent pour ainsi dire que d'une seule, à savoir la justesse du tracé de l'instrument, certaines modalités de facture très prisées de nos jours ne revêtant pour lui qu'un aspect secondaire.

Dans l'esprit de la tradition, bienfacture n'est nullement synonyme de «fignolage». Si elle suppose effectivement cette apparence de propreté du travail, que l'ouvrage soit simple ou très ornementé, elle doit en tous cas laisser transparaître cohérence et justesse dans les proportions et le tracé de l'instrument tout entier. La netteté de l'assemblage n'est alors qu'une conséquence de la conception du travail.

Pour être correct, le tracé de l'instrument doit répondre à deux critères: en effet, lorsqu'il y a interaction entre l'instrument et l'être humain, il faut que l'un des deux présente un plan commun avec l'autre et que, d'autre part, ils se trouvent dans un rapport d'analogie plus ou moins grand, pour que les parties de l'un puissent agir sur l'autre, et vice versa. Comment cette interaction se passe-t-elle entre l'homme et son instrument?

Tout musicien sait que les différentes parties d'un instrument remplissent des fonctions particulières, un peu à la manière des organes dans le corps humain. Ainsi, dans le jeu du luth, la main droite attaque les cordes entre la rosace et le chevalet, tandis que la main gauche raccourcit la longueur vibrante des cordes en prenant appui avec le pouce sur la coulisse du manche: aux deux mains correspondent ainsi deux parties distinctes de l'instrument. Et ne parle-ton pas de la tête et du corps d'un violon ou d'un luth? En anglais, le manche s'appelle neck, le chevalet, bridge. Les exemples pourraient être multipliés qui démontrent que ces dénominations n'existent guère sans analogie avec autre chose, dont il reste à définir les caractéristiques. 
Cette manière de voir est bien illustrée par Robert Fludd, l'un des derniers alchimistes du XVII ${ }^{\mathrm{e}}$ siècle, qui, dans son Utriusque Cosmi Historia (cf. Godwin 1979), représente sous divers aspects le monocorde, l'archétype des instruments à corde. L'une de ces illustrations didactiques (pl. 58) montre un monocorde idéal dont les parties, depuis le bas, sont mises en relation, à savoir: le corps de l'instrument avec les éléments et les astres, le manche avec les hiérarchies angéliques, et la tête de l'instrument avec la Divinité elle-même. Sur une autre planche (pl. 55), on retrouve les mêmes attributions, cette fois-ci en relation avec des parties du corps humain.

Pour décadentes et simplistes que soient parfois les explications de Fludd, elles n'en mettent pas moins en évidence la rigueur plus grande de la cosmologie qui en a été la source. Or, à quelques exceptions près, dont l'ouvrage cité, les livres n'abordaient pas ce qui touche à la pratique relevant de telles conceptions, car celle-ci était liée à la transmission intégrale d'un métier comme celui du luthier. Il ne fait pas de doute que les meilleurs luthiers - Tieffenbrucker, Laux Maler, Hans Frei, Cristofolo Hoch et Stradivari - eurent recours à ces concepts pour construire leurs instruments, ainsi que l'attestent les nombreux tracés directeurs et gabarits laissés par Stradivari ${ }^{1}$. Ces tracés ne toléraient aucune approximation, et ils étaient le fait de maîtres.

\section{Le Trait et son langage}

Qu'est-ce qu'un tracé directeur?

Le tracé directeur donne forme à l'instrument, il le met dans un rapport d'analogie avec le monde et avec l'homme; c'est donc la mesure de cette analogie qui en constitue la perfection.

La genèse de l'instrument par le tracé, devient un processus analogue à la création. Mais avant de passer en revue les modalités de ce processus, voyons quelles sont les conditions qui font du Trait un procédé efficace, et quel en est le langage.

Le Trait suppose un espace qualifié: chacune de ses dimensions et orientations possède une qualité particulière, contrairement au point de vue de la géométrie descriptive moderne, selon lequel la longueur peut être remplacée indifféremment par la hauteur ou la largeur. Rien de tout cela dans l'espace du Trait où il faut reconnaître l'axe vertical, par exemple, comme l'axe Ciel-Terre qui est aussi l'axe vertical de la croix - sans quoi le tracé ne serait que pure convention, ou encore un procédé illusoire.

Le langage du Trait, ce sont les formes qui se ramènent à deux essentielles: le cercle et le carré, associés respectivement au Ciel et à la Terre. Ainsi le monde pouvait-il être représenté par un carré recouvert d'une hémisphère: c'est ce qu'exprime le cœur graphique (fig. 1), qui montre un rabattement dans le plan

\footnotetext{
Voir l'exemple publié par Sons \& Musique pour la Science, Diffusion Belin, p. 76.
} 
du carré de base des deux projections sur deux plans verticaux orthogonaux de la coupole qui le surmonte. Dès la Renaissance, cette représentation symbolique du monde a été dépréciée; prétendument le fruit de la naïveté et de l'ignorance, elle a dû céder la place à des considérations strictement astronomiques fondées sur le rejet de son domaine de significations.
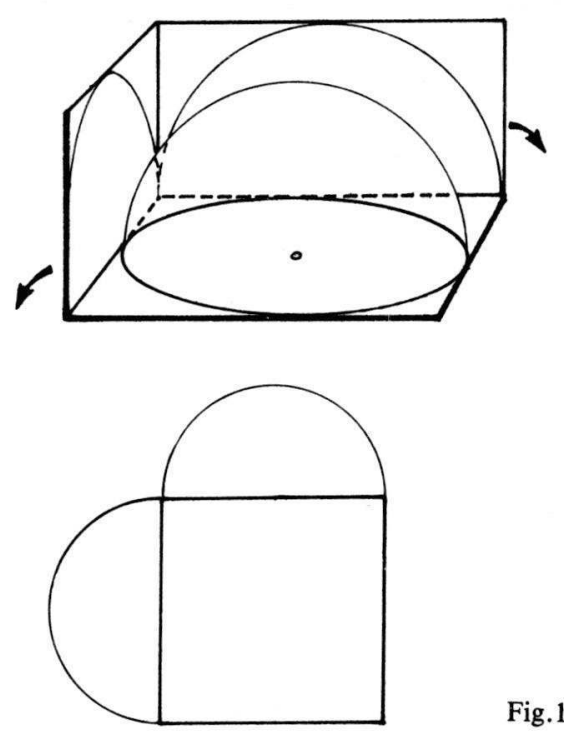

Fig.1

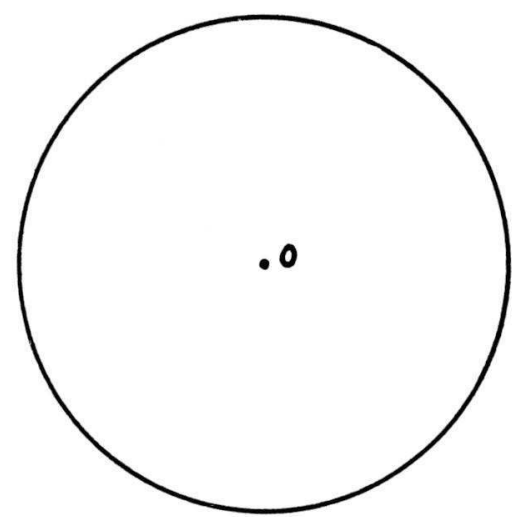

Fig. 2

Par ailleurs, le caractère d'indifférenciation et de non-manifestation, voire de potentialité, est aussi associé au cercle et davantage encore à son centre qui n'est manifesté que par sa "périphérie», sa circonférence. Les anciens avaient, en effet, une perception des formes toute différente de la nôtre: en regardant le cercle, ils voyaient le centre. Les modernes, en revanche, ne lui accordent plus cette signification, dans la mesure où ils considèrent tous les points de la courbe plutôt que le centre qui l'a engendrée. Le centre, tout comme le cercle, n'orientent pas l'espace; ils en sont la source.

Ajoutons que ce regard posé sur les courbes a l'avantage de permettre d'y voir autre chose que des accidents de forme imprévisibles et inintelligibles, et de les mémoriser plus facilement par le fait de les comprendre.

Le carré, en revanche, est le lieu de la norme, de l'accession au monde sensible d'un concept - rien ne pouvant être mesuré sans l'aide de segments de droites orthogonales. 
Les deux notions du cercle et du carré étant très sommairement posées ${ }^{2}$, il s'agit d'aborder la question de la genèse. Dans le cas du tracé d'un instrument, c'est un processus fort complexe. Pour l'illustrer, il faut avoir recours à un tracé apparemment plus simple, qui est celui du calice. Ce dernier a une valeur de représentation universelle, qui explique l'importance que le Moyen Age y attachait (cf. le Graal).

Voici la manière dont on peut tracer le calice:

1. On choisit un point d'origine $O$, puis on trace autour de lui une sphère: voilà la représentation de la potentialité pure avec sa source (fig. 2). Jusqu'ici, tout est possible, et c'est bien ainsi que commence tout tracé. L'espace n'est pas encore qualifié.

2. Cette sphère est traversée en son centre $O$ par un axe vertical qui implique déjà un plan horizontal défini par les deux droites qui lui sont perpendiculaires et qui sont perpendiculaires entre elles; ces droites peuvent passer également par le centre $O$ de la sphère. Jusque-là, tout reste possible, mais l'espace est néanmoins déjà qualifié (fig. 3).

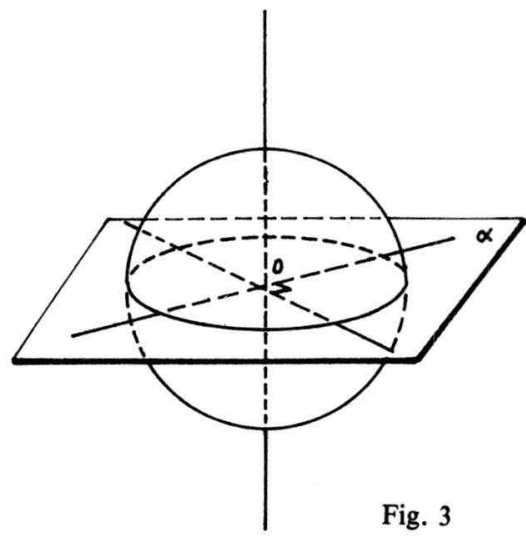

Fig. 3

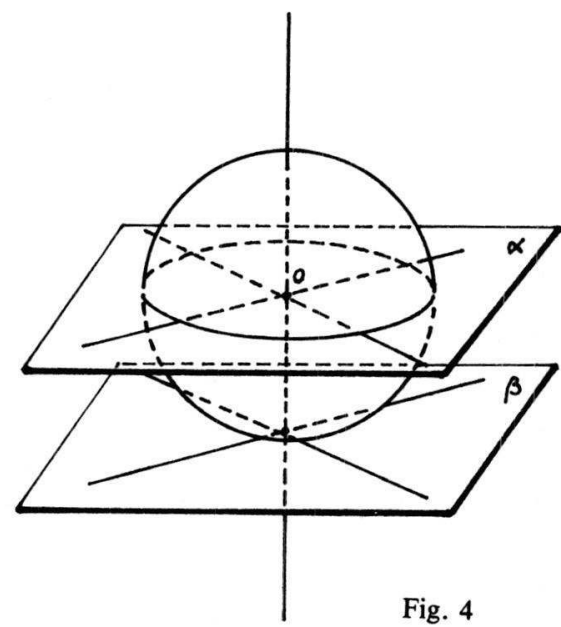

Fig. 4

2 A l'appui de cette manière de voir, on pourrait citer in extenso le recueil d'articles de Titus Burckhardt (1987), qui explicite avec plus de détails que ne peut le faire le présent article, la vraie nature de la figure géométrique et la manière dont ses variantes revêtent son sens sans jamais le rendre trivial. 
3. En-dessous de ce plan $\alpha$ et parallèlement à lui, on peut tracer un plan $\beta$ à une certaine distance d' $\alpha$, par exemple égale au rayon de la sphère (fig. 4). Toute est encore possible, mais l'espace est maintenant normé par la distance séparant les deux plans $\alpha$ et $\beta$.

4. Maintenant, le cercle équatorial de la sphère se projette perpendiculairement sur le plan $\beta$ en un cercle de même diamètre que la sphère (fig. 5).

5. Ce cercle devient le lieu des centres de sphères égales à la première, décrivant le pied du calice par leur intersection avec un plan pivotant sur l'axe vertical et passant par leur centre; ce pied est limité en bas par un plan situé en-dessous de $\beta$ et à une distance égale à celle qui sépare $\beta$ de $\alpha$ (fig. 6).

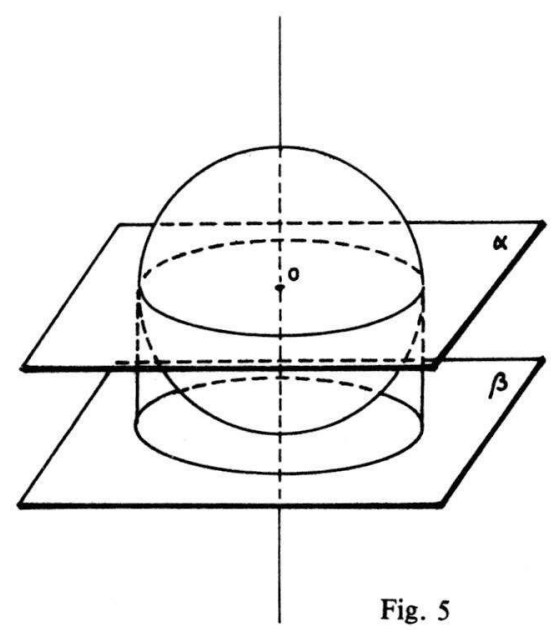

Fig. 5

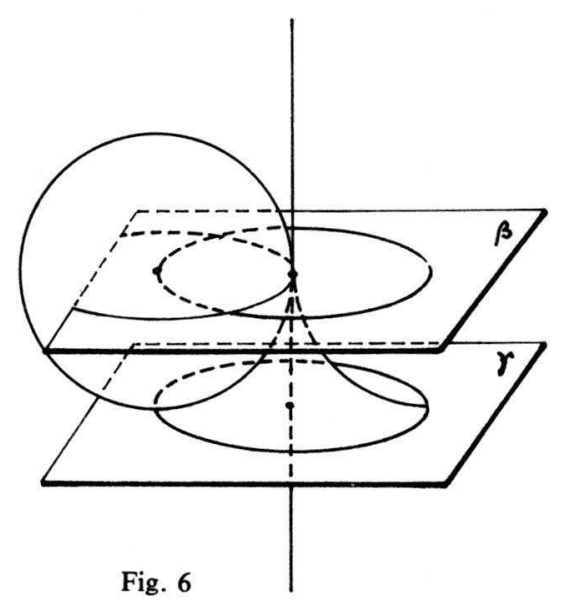

Fig. 6

On obtient donc un calice limité par trois plans superposés, le plan $\beta$ étant souvent souligné et dédoublé, en orfèvrerie, par une petite sphère supplémentaire marquant le passage du centre des sphères à la périphérie sur le plan $\beta$ '; ce passage n'est vraiment explicte que sur le plan $\gamma$ (fig. 7).

En d'autres termes, un point se manifeste par une sphère apparaissant autour de lui; cette sphère se projette sur un plan qui lui est inférieur, mais tangent. Sur ce plan, des sphères semblables à la sphère d'origine deviennent "périphériques", tout en conservant une tangente à l'axe vertical passant par le centre initial $O$. Enfin, sur le plan $\gamma$, la trace des sphères est devenue complètement périphérique.

Ainsi s'exprime un aspect du processus créateur en langage géométrique, qui équivaut en fait à un décentrage progressif. Ce dernier est le principe même de toute manifestation, et nous reviendrons plus loin sur le rapport qu'il entretient obligatoirement avec le monde sensible. 
Le tracé dont nous venons de passer en revue les principales étapes, aurait pu être différent pour montrer un autre aspect de la réalité, mais sans perdre pour autant son sens. Le lieu du centre des sphères décrivant le pied du calice, par exemple, pourrait être autre chose que la projection orthogonale de la sphère initiale sur $\beta$, soit sa projection centrale à partir d'un point $\mathrm{A}$ situé sur l'axe au-dessus de la sphère, ce qui supposerait une antériorité de A qui serait alors le point de vue originel engendrant le point $O$. Le pied du calice s'en trouverait agrandi par rapport au tracé décrit ci-dessus (fig. 8).

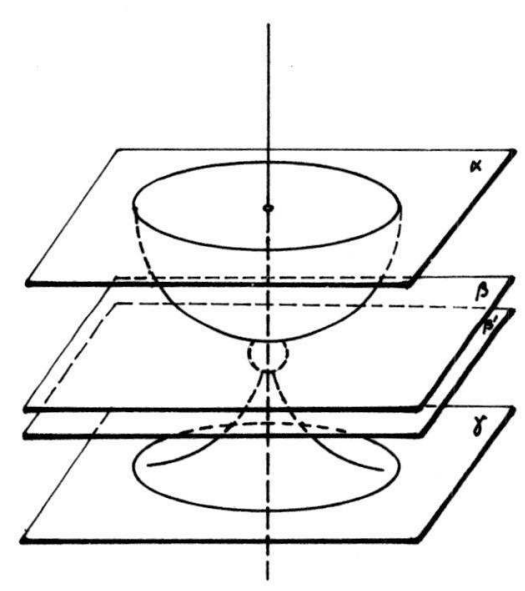

Fig. 7

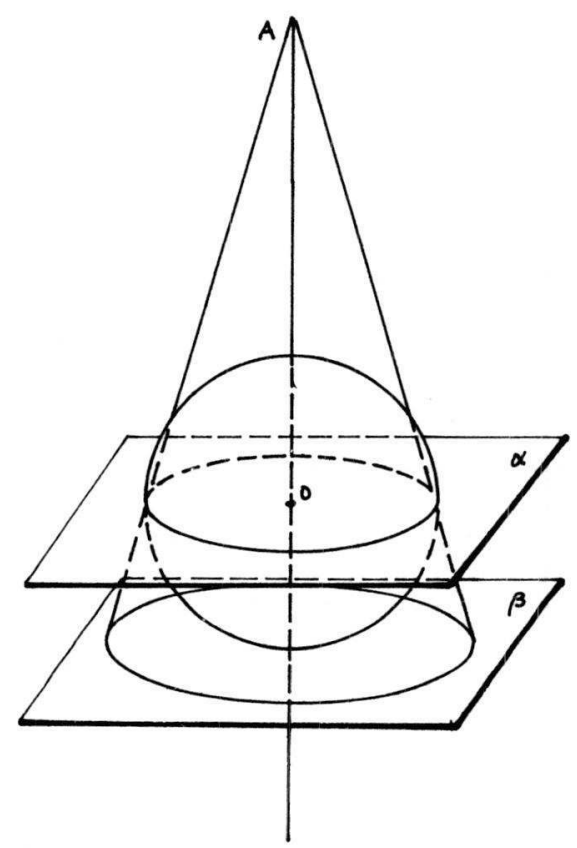

Fig. 8

Au lieu de décrire le pied au moyen d'une révolution de sphère, on pourrait aussi le faire par une translation sur le carré circonscrit à la projection de la sphère initiale sur $\beta$, ce qui donnerait un pied carré, comme on le reconnaît avec des complications de formes sur de nombreuses pièces d'orfèvrerie (fig. 9 et 10).

Un même modèle de tracé comporte une quantité de variantes, et l'on peut dire que la réalité spirituelle que l'on veut exprimer ne prend vraiment forme que lorsque toutes les possibilités auront été tracées, ce qui est matériellement irréalisable. 


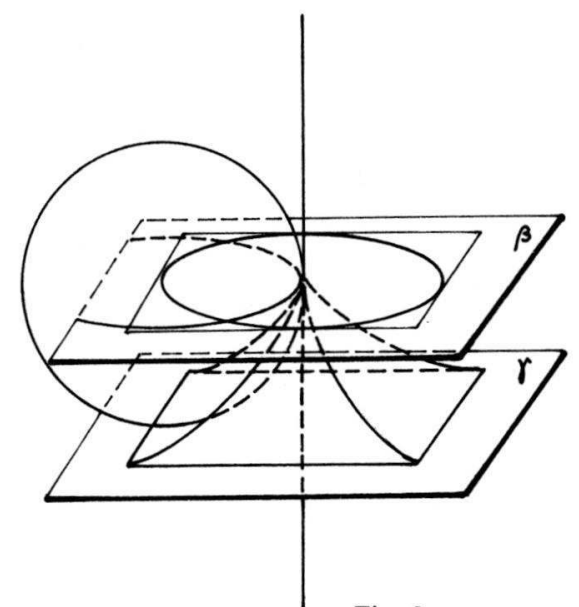

Fig. 9
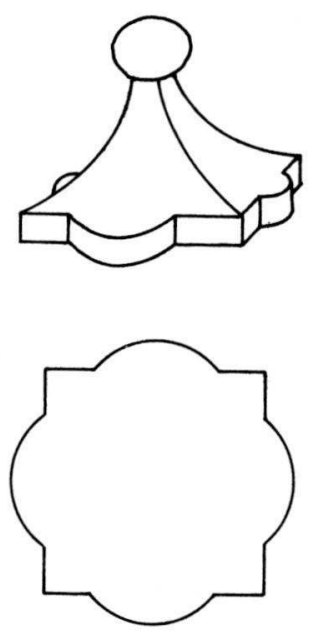

Le tracé que nous venons de faire est un tracé directeur, établi conformément au fondement cosmologique ${ }^{3}$ et en respectant certains ordres de manifestation d'un principe unique.

A elle seule, une version de ce tracé ne saurait rendre compte de la réalité tout entière, puisque celle-ci n'est exprimable que par une pluralité indéfinie de variantes. Mais ce tracé n'en repose pas moins sur une vérité doctrinale formulée en termes théologiques. Comme le dit si bien Titus Burckhardt (1976: 11):

«Ceci exprime [...] la nature de l'art sacré: en retraçant un prototype sacré, qu'il adapte à telles conditions matérielles, l'artiste s'identifie lui-même à ce prototype, en l'extériorisant conformément aux règles transcrites, il en réalise l'essence».

3 Il faut relever, et ceci est essentiel, que cette conformité ne peut avoir de sens sans une convention très clairement admise par l'exécutant de l'œuvre et ses utilisateurs. Cette convention implique, entre autres, qu'il n'y a pas identité de nature entre la réalité exprimée et l'œuvre, mais seulement similitude dans les rapports internes. Voir dans une œuvre la Vérité est une attitude idolâtre. Il est cependant légitime de reconnaître dans une œuvre d'art, avec les limitations inhérentes à la forme d'art en question, un aspect de la Vérité. Le fabricant d'un instrument de musique devra donc se conformer aux mêmes conventions, ou à des conventions compatibles avec celles qui règleront son utilisation; sinon, étant produit et utilisé dans deux contextes différents, l'instrument perdra sa valeur de représentation. C'est exactement ce qui rend difficile l'utilisation d'instruments anciens et même l'exécution de musique ancienne dans le contexte moderne, dont les présupposés sont nécessairement fort différents. 


\section{Le Trait dans la lutherie}

Le tracé du calice est remarquable, car il est le même que celui de la trompe, instrument par excellence, à côté du luth, des anges dans l'iconographie chrétienne médiévale. Le bocal de l'embouchure de la trompe correspond au bocal du calice, la sphère de liaison correspond à la «pomme» de la trompe d'église, et le pied, au pavillon.

Les instruments à cordes, et surtout le luth, ne font pas exception aux règles dégagées à propos du tracé du calice. Chaque type d'instrument possède son propre tracé, les proportions mises en jeu relevant de l'inspiration de celui qui y recourt. C'est ainsi qu'un tracé peut aboutir ou non: chaque phase du tracé ayant une signification précise, le choix d'une proportion peut, à un moment donné, en assurer ou en compromettre la suite, et il arrive qu'une étape s'avère impossible ou que la forme finale se révèle être totalement aberrante ${ }^{4}$.

Kevin Coates illustre ce phénomène à l'aide de nombreux exemples pris sur des instruments anciens - violes, violons, guitares, cistres, luths et théorbes - mais sans indiquer comment il a identifié les centres de courbure de ses exemples et, surtout, sans dégager clairement les principes de tracé.

Le présent article se propose ainsi de montrer de quoi il retourne et aussi d'en comprendre l'enjeu. Le premier point laissé sans explication par Coates se résoud facilement. Pour trouver les centres de courbure d'un trait, il suffit de lui tracer des tangentes à intervalles réguliers; puis, au point même de tangence, on leur trace une perpendiculaire. Les points d'intersection des perpen-

4 On peut déjà voir par cet exemple que, dans une œuvre d'art, ce que l'on peut y lire importe beaucoup plus que l'habileté avec laquelle elle est exécutée. Dans de tels tracés tout est, non seulement signifiant, mais aussi rétroactif, en quelque sorte. C'est dire que, en se conformant à un archétype et en l'actualisant, l'artisan peut le faire avec une transparence plus ou moins grande. Si l'artisan pose des obstacles à l'action de la grâce et de l'inspiration divine, son œuvre va se trouver entachée d'erreurs, tout comme un texte peut énoncer des choses fausses; et ces erreurs auront une action sur ceux qui en deviendront les utilisateurs, créant par là un désordre insidieux dans le monde, d'autant plus que les utilisateurs de l'œuvre ne seront pas forcément qualifiés pour en déceler les vices. C'est, aux yeux de l'artisan traditionnel, une des raisons de la nocivité de l'art moderne. L'art du Trait dans le compagnonnage a donc un caractère d' "aventure» spirituelle qui s'avèrera fructueuse ou non, une fois l'œuvre achevée, car son usage en montrera les qualités ou les défauts: une cathédrale au tracé régulateur correct selon les critères traditionnels sera belle de sa participation à la splendeur incréée, et accessoirement sera stable de la stabilité la plus matérielle; un instrument bien dessiné sonnera d'un son ineffable et sera donc commode à jouer et résistera bien à la tension des cordes. Cela est la meilleure démonstration de l'analogia entis pour l'artisan: c'est une corrélation qui ne peut faire de doute que pour celui qui ne veut pas l'expérimenter. En ce sens, le travail est le canal par lequel l'artisan confirme sa foi. On remarque également que l'habileté n'est pas traditionnellement un critère. "L'ouvrier habile n'a jamais suffi à faire le bon ouvrier», dit le compagnonnage. Si une œuvre est vraie, il se dégage toujours d'elle une beauté proportionnelle à son degré de vérité, sans rapport obligatoire avec l'habileté mise en œuvre pour son exécution. C'est ce qui explique que les ouvrages anciens, sous une apparente maladresse d'exécution, manifestent une splendeur plus grande que des ouvrages plus habiles, «car les enfants de ce monde-ci sont plus avisés avec leurs semblables que les enfants de Lumière» (Lc, 16,8). 
diculaires sont des centres de courbure. Cette méthode n'est pas très précise, mais elle suffit en tout cas pour situer un centre de courbure sur un instrument dont la forme peut ne pas avoir été exécutée avec une parfaite exactitude ou avoir subi de légères déformations lors de réparations ou simplement à cause de la rétraction du bois au vieillissement, ou davantage encore à cause de modalités de construction qui occultent au premier examen la forme initiale ${ }^{5}$.

Le recours à ces proportions simples n'est d'ailleurs pas l'apanage des constructeurs d'instruments occidentaux. Il suffit, pour s'en convaincre, d'examiner un pipa chinois ou une cobza roumaine, sans parler de la «guitare-lune» (yueqin) de la Chine du Sud, dont la table est circulaire.
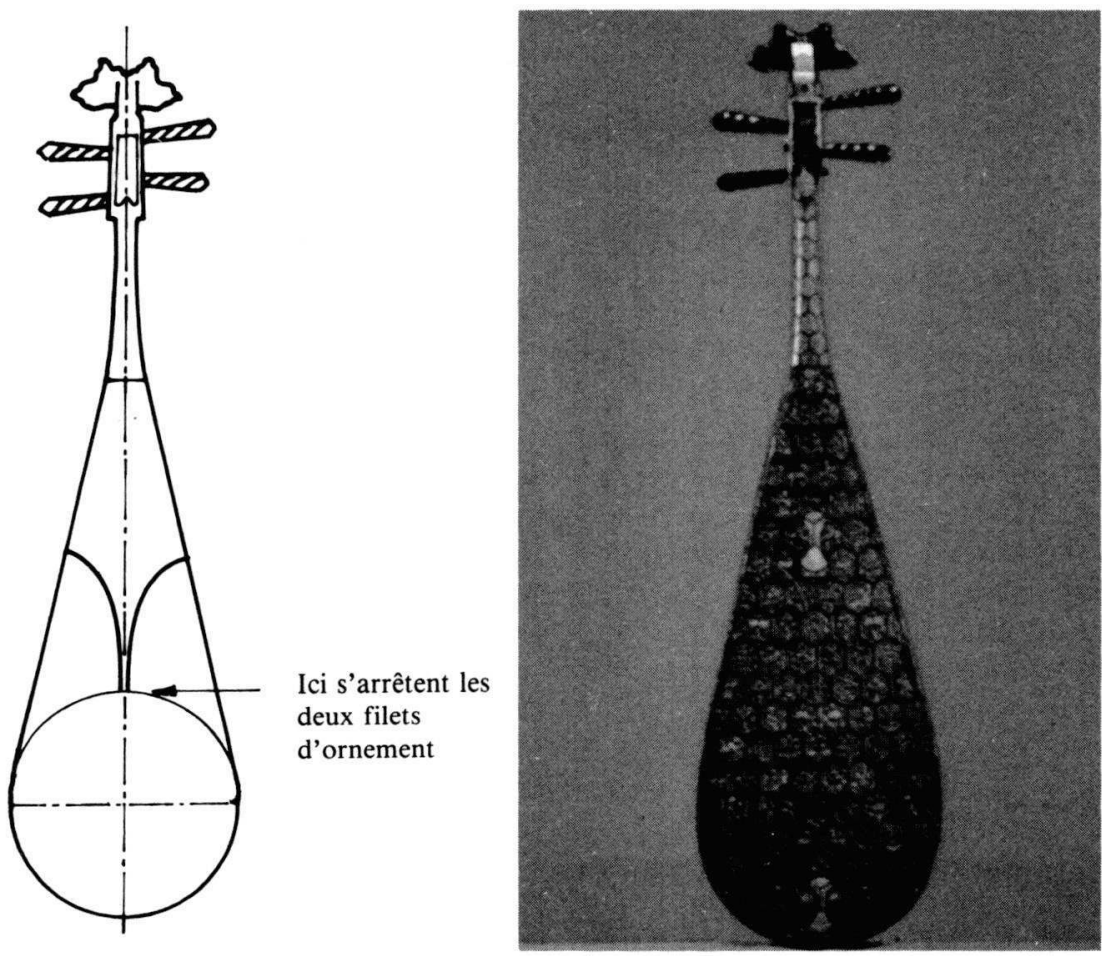

Fig. 11: Pipa, du Metropolitan Museum of Art.

5 Ce point mérite d'être souligné. Dans l'instrument fini, il se trouve souvent qu'une partie ou tout du tracé n'apparaissent plus directement à l'observateur, sans pour autant avoir diparu. Le luth offre un exemple significatif: un grand nombre de luths occidentaux et la quasi-totalité des luths arabes ont une coque à révolution circulaire (si l'on abstrait les côtes anguleuses sur le luth occidental, ce sont alors leurs arêtes qui se trouvent sur un volume engendré par la révolution d'un gabarit autour d'un axe). Si la table de l'instrument ne passe pas par cet axe, la forme de cette table ne fera pas apparaître la surface génératrice de la coque, mais une déformation plus ou moins accentuée de celle-ci. Il n'en demeure pas moins que, pour n'être plus 
La forme du pipa est un demi-cercle surmonté d'un triangle isocèle (fig. 11); celle de la cobza est à peine plus compliquée (fig. 12). Une étape ultérieure dans la complexification est illustrée par le luth de forme ovoïde simple (fig. 14), décrit par Arnault de Zwolle (cf. 1972).

La plus élémentaire de toutes ces formes nous introduit directement à la notion de décentrage qui va servir de justification au tracé, car si le tracé est sans rapport avec la production du son, à quoi bon tracer?

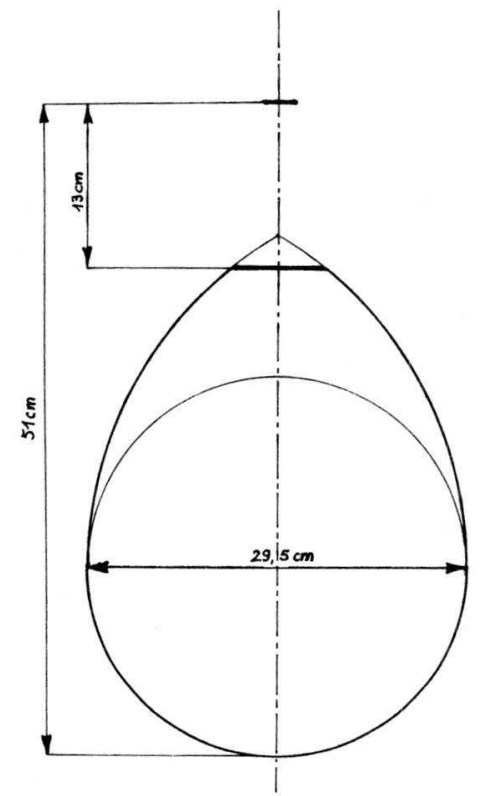

Fig. 12: Cobza (Roumanie).

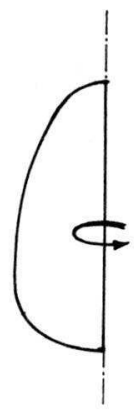

Fig. 13

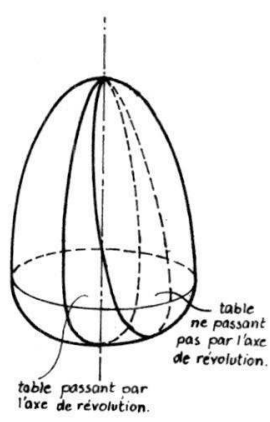

\section{Une correspondance physique}

Son et tracé sont indissociable. Il a déjà été dit qu'aucun phénomène ne se manifeste sans qu'il y ait décentrage, de quelque manière que ce soit. Par exemple, un disque parfaitement centré et animé d'un mouvement de rotation

directement lisible, cette surface continuera à jouer dans l'instrument un rôle capital (fig. 13). C'est pourquoi une analyse de forme requiert beaucoup de soin, et de très nombreux cas rendent l'approche strictement analytique d'un instrument ancien des plus hasardeuses, par le fait que l'analyste perd rapidement de vue les points d'articulation nécessaires à son raisonnement. Il s'ensuit que la copie d'instruments anciens, et même d'œuvres d'art en général, est un exercice difficile, car force est de constater que toute œuvre authentique est en ce sens unique. 


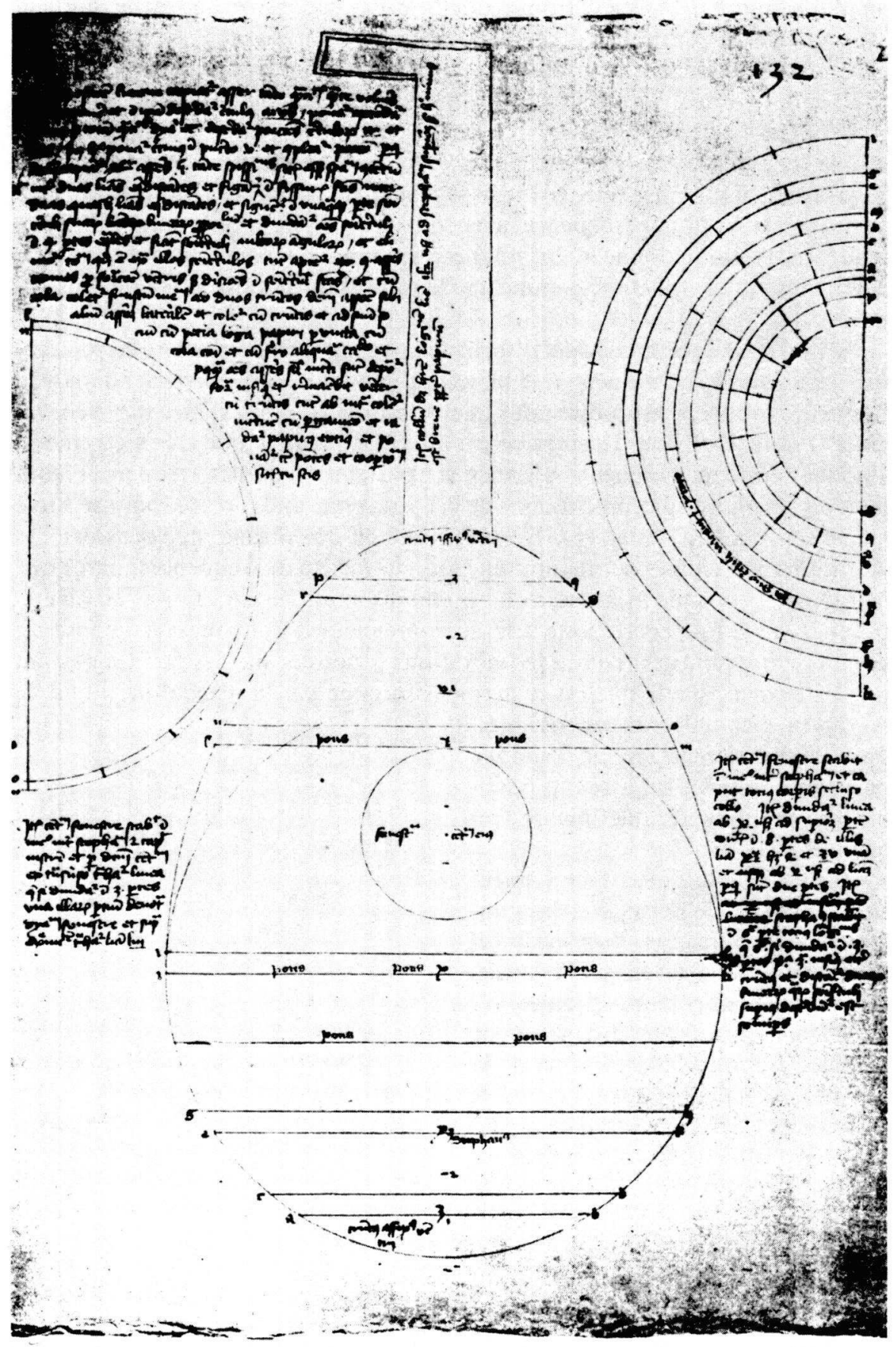

Fig. 14 
semblera immobile. Qu'il se trouve ailleurs qu'en son centre une tache, que son axe soit légèrement décentré, le phénomène se manifeste aussitôt à l'observateur: la périphérie semble osciller, le mouvement est devenu perceptible.

Il en est de même pour l'instrument à corde. Prenons le cas du yueqin ou du banjo, tous deux de forme circulaire. Leur principe étant d'adjoindre à la source qu'est la corde vibrante une surface apte à répondre aux fréquences diverses que lui impose cette corde, il se trouve que la table de bois ou de peau ne peut émettre qu'une fréquence, avec son cortège d'harmoniques spécifiques, à surface et tension données. Or, pour être «amplifiée» par cette table, la corde devra l'être pour toute une gamme de fréquences qu'impose le jeu de l'instrument.

Si l'on pose donc le chevalet d'un instrument de forme circulaire en son centre, il ne pourra théoriquement émettre qu'une seule note correspondant à sa fréquence propre, c'est-à-dire pour une vibration ayant un ventre sur le chevalet. Par contre, si l'on décentre ce chevalet, ne serait-ce que très légèrement, aussitôt plusieurs longueurs d'ondes et, partant, plusieurs fréquences vont pouvoir répondre aux sollicitations de la corde pour une surface soumise à une même tension (fig. 15 et 16). De plus, avec ce décentrage, apparaissent des vibrations non axiales permettant une grande variété de fréquences, parce que leur longueur d'onde locale sera plus ou moins modifiée par rapport au diamètre de la table (qui correspond à la demi-longueur d'onde maximale possible sur un tel instrument). Toutes les vibrations localisées sur des bandes passant par le chevalet, sur lesquelles ce dernier n'occupe pas la place d'un nœud de vibration, seront désormais possibles.

Par simple décentrage de l'unique possibilité initiale, on a rendu possible une multiplicité indéfinie de fréquences.

Il ne s'agit là que d'une manière très simplifiée de considérer les choses. Mais comme le démontre cet exemple, un tracé directeur va consister essentiellement à organiser le décentrage, par des modifications de surface, du point d'application de la vibration de la corde sur un corps de résonance. La manière dont ce décentrage sera effectué va déterminer les fréquences de la corde qui seront réémises par la caisse de l'instrument, en autorisant ou même en favorisant certaines d'entre elles, tout en interdisant peut-être d'autres. Tout l'art
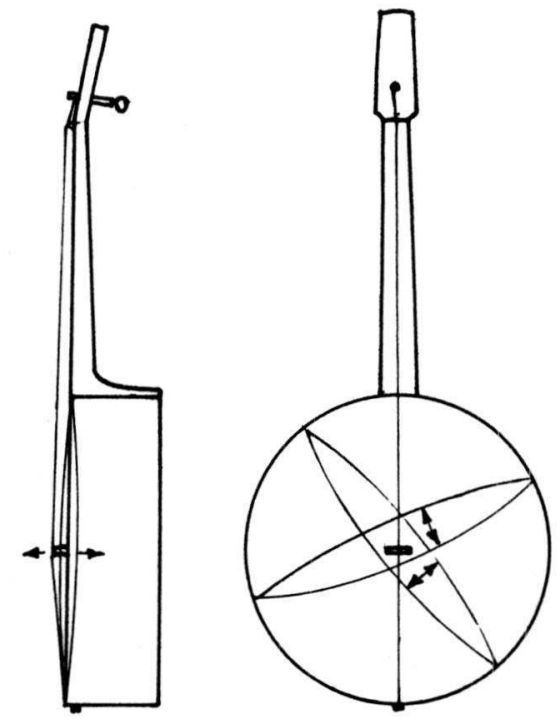

Fig. 15 

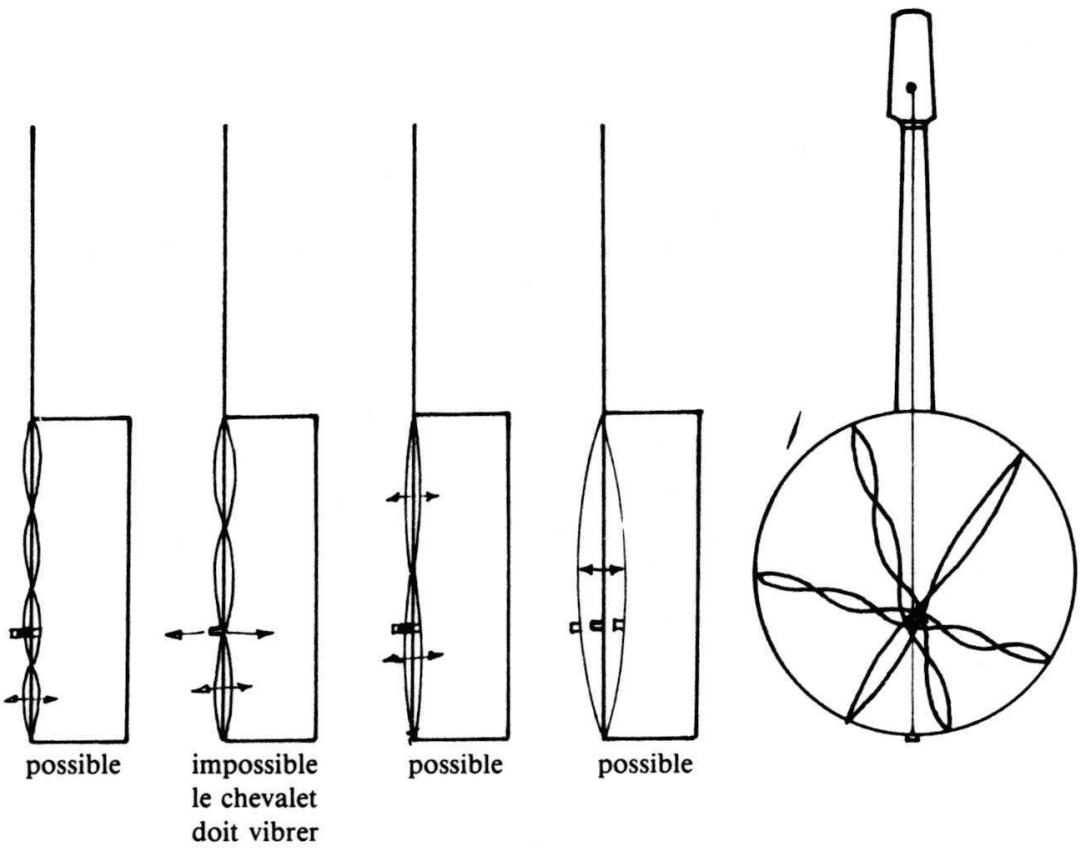

Fig. 16

consiste à exploiter la corrélation qu'il y a entre le décentrage géométrique et le déséquilibre périodique qu'est le son.

\section{Le modèle et ses extensions}

Mais tout cela ne donne toujours pas le modèle du tracé des instruments: voilà le second point laissé en suspens par Kevin Coates. Il s'agit d'une représentation dynamique du passage de l'Unité incréée, non manifestée, ou déjà manifestée, à la multiplicité. Cette dernière est ordonnée de telle sorte qu'elle semble, pour ainsi dire, chanter à la louange de cette Unité incréée dont elle procède.

Dans le cas de l'instrument à caisse circulaire, il n'y a qu'un centre. Les instruments à description plus complexe font apparaître plusieurs centres secondaires. Ainsi le point initial perd-il sa qualité physique de centre du mouvement pour ne conserver que sa qualité de source géométrique. Sa fonction physique est alors transférée avec des proportions diverses aux centres secondaires. Mais comment ces centres secondaires préservent-ils leur fonction lorsque les surfaces qu'ils régissent sont plus ou moins grandement abouchées avec d'autres surfaces centrées? 
Eh bien, dans ce cas, c'est la fraction de périphérie leur correspondant dans la forme globale de l'instrument, qui leur conserve ce rôle que l'on pourrait expliquer par un effet d'équidistance. C'est précisément par cette fraction que l'on va pondérer un centre de courbure par rapport aux autres.

Cela confirme le fait qu'un centre géométrique initial dont rien du périmètre ne demeure dans la forme finale, ne joue plus de rôle physique dans la vibration: il l'a entièrement délégué aux centres secondaires qu'il a engendrés. En ce sens, un centre de courbure se trouve cautionné par le reste de son périmètre demeuré dans la forme finale par le fait que, par équidistance de sa périphérie qui le rappelle à la position d'équilibre, un tel point est susceptible de la plus grande amplitude de vibration.

Pour illustrer ces propos, prenons un exemple encore peu complexe de tracé du luth Renaissance (fig. 17). On trace un cercle de centre $O$ et de rayon $R$. En son centre passent l'axe vertical AZ et l'axe horizontal IJ. En prenant I et J comme centres, on trace une vesica piscis de sommets $\mathrm{A}$ et $\mathrm{Z}$, dont le rayon de courbure est $R \sqrt{2}$. De même, on trace une autre vesica horizontale de sommets I et $\mathrm{J}$ avec même rayon de courbure à partir des centres $\mathrm{A}$ et $\mathrm{Z}$. Sur les diagonales $\mathrm{BD}$ et $\mathrm{CE}$, on raccorde les arcs de centre $\mathrm{A}$, I et $\mathrm{J}$ par deux cercles de centres $\mathrm{H}$ et $\mathrm{H}^{\prime}$ et de rayon

$$
\mathrm{r}=\frac{2 \mathrm{R}}{3}(2-\sqrt{2})=\frac{1}{3} \overline{\mathrm{FG}}
$$

On a ainsi tracé par une séquence de courbures convexes une forme de luth.

Avec des centres de courbure comparables et un cercle de rayon convenablement choisi au pôle supérieur, mais des courbures concaves centrées sur l'axe horizontal, on aurait une guitare baroque ou un violon (sans les coins des C) (fig. 18 et 19). Le pôle supérieur peut être un cercle ou même, surtout dans la facture postérieure à 1800 , être comme les cercles inférieurs, dédoublé horizontalement.

Parvenu à ce point, il est nécessaire de préciser en quoi un tel tracé diffère d'une simple construction géométrique et ce que ceci implique. Une construction géométrique est une séquence d'opérations s'articulant les unes avec les autres, et à laquelle préside la logique discursive: il n'y a pas d'autres liens entre les étapes que la force contraignante du raisonnement, et éventuellement certains choix secondaires lorsqu'on se trouve au carrefour de plusieurs possibilités. Un tel choix peut d'ailleurs faire l'objet de rétractations s'il s'avère peu avantageux.

En ce qui concerne le Trait, c'est tout autre chose. Un tracé n'est guidé que par une inspiration, reconnue de nature angélique, puisque ce sont les anges qui président aux fonctions cosmiques. Cela ne veut pas dire que le Trait désavoue le rationnel; il le contient tout en le dépassant. La logique est la moindre de ses nécessités, mais c'en est paradoxalement aussi une.

Il s'ensuit que d'une part, le Trait ne peut s'exercer sans une disposition spéciale: c'est ce qui a été appelé plus haut la grâce d'état de l'artisan. D'autre part, cela ne peut avoir lieu sans que se produise une émotion particulière, comme un trouble, qui n'a rien à voir avec la confusion, et qui fait entrevoir 


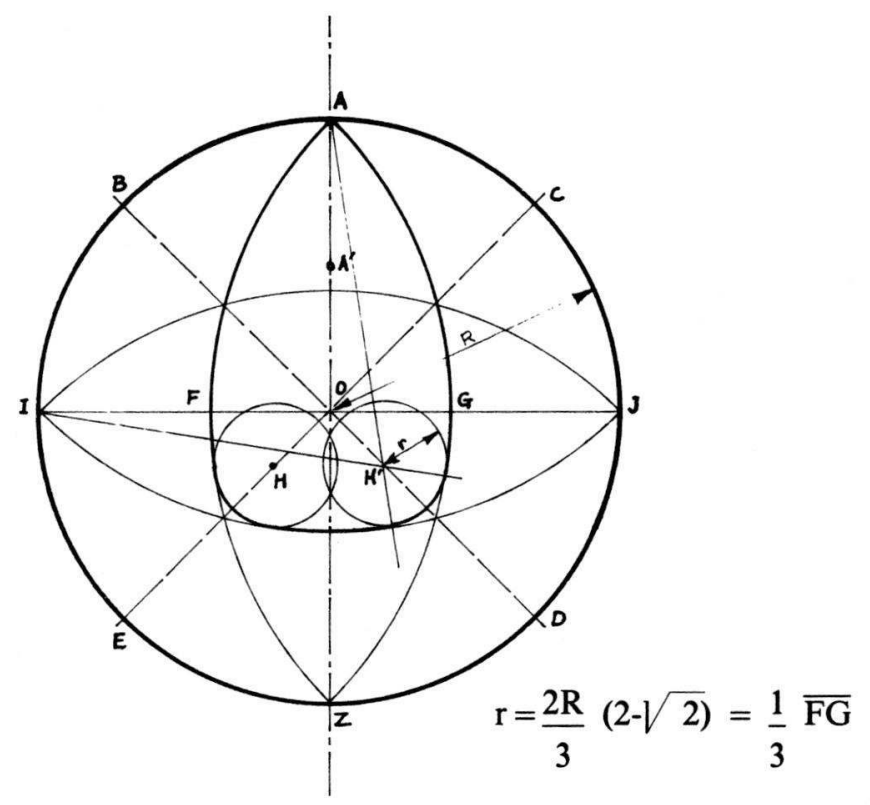

Fig 17

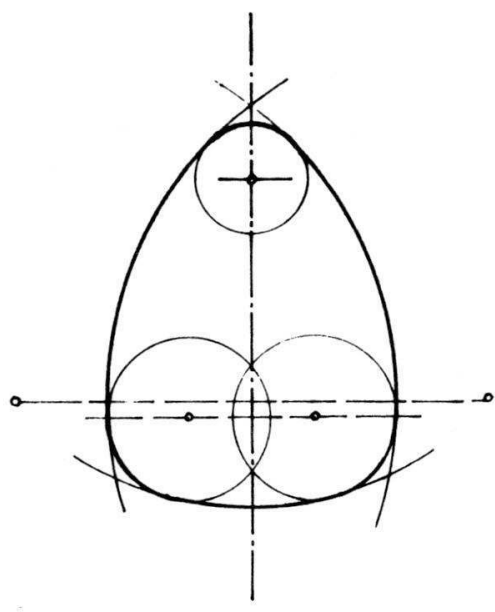

Luth

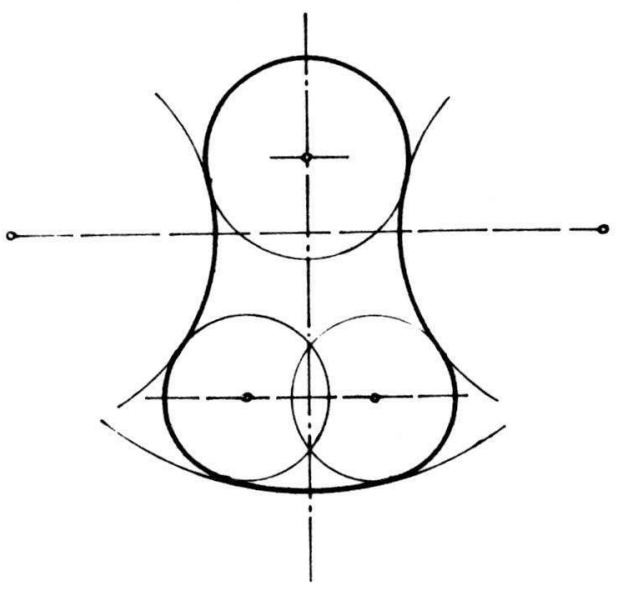

Violon et guitare

Fig. 18 


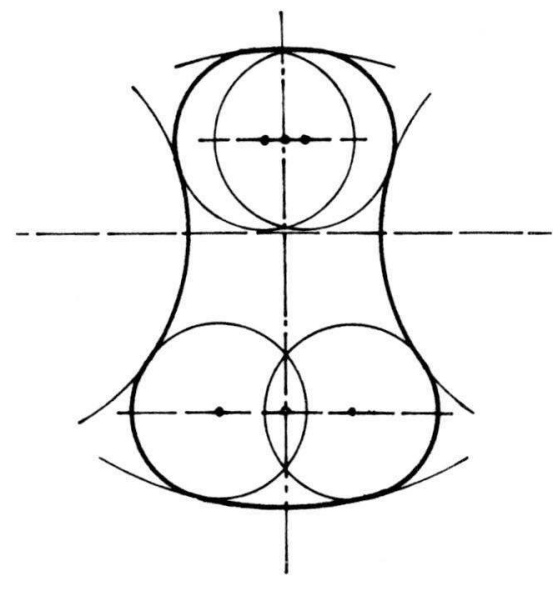

Fig. 19

des choses que l'on ne peut percevoir dans l'état ordinaire. Ce n'est pas comparable à un sentiment, et pourtant engendre une certitude beaucoup plus grande que toute opération des sens.

On comprendra alors que ce procédé ne saurait obéir aux impératifs de reproductibilités qui servent de fondement à toute méthode scientifique moderne. Telle est la nature du Trait: c'est ainsi qu'il se situe audessus d'une combinaison de disciplines scientifiques, et qu'il peut avoir des effets qui dépassent complètement le simple calcul, si habile soit-il.

Leur apparente complexité fait que les instruments occidentaux semblent être faits de formes plus accidentelles que leurs homologues orientaux. Mais il n'en est rien, surtout pour les instruments portés par l'instrumentiste en cours de jeu. On pourrait en dire autant de bien des clavecins qui devaient impérativement posséder cette structure, limités qu'ils étaient dans le choix des cordes, les cordes filées étant en effet systématiquement évitées.

Ce n'est par contre plus le cas du piano, dont la forme est essentiellement liée à des questions d'encombrement, les problèmes sonores étant résolus par l'emploi de cordes plus ou moins lourdes, sans que le fonctionnement général de l'instrument soit tenu en compte. De plus, sa géométrie s'est affadie par la disparition de l'axe vertical dans son tracé, ainsi que par l'absence d'une symétrie ou d'une dissymétrie significative.

Ce procédé par tâtonnements a été rétroactivement appliqué à tort à la facture moderne des instruments anciens qui relèvent d'une toute autre optique, les rendant par là incompréhensibles et de plus en plus difficilement utilisables.

Les formes à plusieurs centres des instruments de facture traditionnelle ont été envisagées d'une manière simplifiée, car ces considérations ne valent que pour une forme non barrée. A toute table d'instrument sont en effet adjointes une ou plusieurs barres à l'intérieur, ou encore à l'extérieur dans le cas particulier du chevalet collé. Ces barres modifient considérablement la fonction dévolue aux centres par la forme: elles inhibent plus ou moins fortement leur mouvement potentiel pour le répercuter, le répartir. Mais tout cela appelerait un long développement.

Afin de situer ces phénomènes dans leur juste contexte, observons qu'entre considérations d'ordre physique et artifices (au sens d' "exercices de l'art»), il est vain de tenter d'établir une quelconque parité ou de n'y voir que des 
concordances: il s'agit irréductiblement de deux plans différents. L'art ne se réduit pas à la manière d'utiliser des phénomènes physiques, sans pour autant entrer en contradiction avec eux.

L'art doit en tout état de cause représenter le plus clairement possible des rapports internes, mais sans jamais cacher la relation à la source véritable du monde manifesté: c'est là, en somme, la convention indispensable à l'exercice de l'art. Envisagé de cette manière, le système fermé cher aux sciences physiques serait rélégué au rang de parodie, et le substituer à l'art serait une forme de superstition.

En conclusion, un exemple pratique commun à plusieurs métiers illustre ce propos: il s'agit du rapport qu'il y a entre l'ouvrage fini et les déchets que le travail a occasionné.

L'ouvrier dispose au départ d'une masse relativement dépourvue de qualifications, encore qu'il lui incombe d'être attentif à certaines. Cette masse contient potentiellement toutes les formes possibles, et il doit en déterminer une en exerçant un pouvoir séparateur, se mettant ainsi dans une situation comparable à Celui qui use de l'épée double de l'Apocalypse. Cela signifie qu'il prédétermine ce qui, dans cette masse, deviendra l'objet, ce qui deviendra déchet, et par son travail il va mettre dans un état de désagrégation irréversible ce qui doit être déchet, tout comme le fondeur casse son moule. Par cet acte, l'ouvrage entre dans la manifestation.

Si ce processus est irréversible, l'artisan n'en est pas moins appelé à le considérer dans son ensemble: les déchets, tout comme l'objet façonné, jugeront son travail. C'est ainsi qu'un sculpteur aura d'autant mieux travaillé que ses copeaux seront beaux et qu'il aura produit peu de poussière (contrairement aux machines modernes). A ce propos, on relira avec profit la légende de saint Éloi, car il en est de même de l'économie du geste.

Ces critères, par la force des choses, ne sont appréciables que par l'homme du métier; ils échappent généralement au futur utilisateur de l'ouvrage, ce qui est souhaitable, car ce sera pour lui une nouvelle materia en quelque sorte "purifiée» par le fait qu'il n'en connaît pas toutes les modalités de facture. Seuls les défauts ou qualités constatables par lui entreront en ligne de compte. C'est une des raisons pour lesquelles il n'est normalement pas souhaitable qu'un même homme soit auteur et utilisateur de son œuvre.

\section{Bibliographie}

ARNAULT DE ZWOLLE Henry

1972 Un traité d'Henry Arnault de Zwolle et divers anonymes. Kassel/Basel/Tours/London: Bärenreiter.

BURCKHARDT Titus

1975 Principes et méthodes de l'art sacré. ?: Dervy Livres.

1987 Aperçus sur la connaissance sacrée. Milan: Arché.

COATES Kevin

1985 Geometry, proportion, and the art of lutherie. Oxford: Clarendon.

GODWIN Jocelyn

1979 Robert Fludd. London: Thames \& Hudson. 